\title{
Efeito da Densidade de Estocagem no Desempenho de Rã-Touro (Rana catesbeiana) em Recria ${ }^{1}$
}

\author{
Alex Poeta Casali ${ }^{2}$, Onofre Maurício de Moura ${ }^{3}$, Richélita do Rosário Brito Mendes ${ }^{4}$, Valnir de \\ Meneses Campos ${ }^{5}$
}

\begin{abstract}
RESUMO - Este trabalho foi realizado com o objetivo de avaliar quatro densidades de estocagem na recria de rã-touro em mini-baias. As densidades estudadas foram: D1: 75 rãs $/ \mathrm{m}^{2}$ (inicial) e $35 \mathrm{rãs} / \mathrm{m}^{2}$ (final); D2: $105 \mathrm{rãs} / \mathrm{m}^{2}$ (inicial) e 50 rãs $/ \mathrm{m}^{2}$ (final); D3: $125 \mathrm{rãs} / \mathrm{m}^{2}$ (inicial) e 60 rãs $/ \mathrm{m}^{2}$ (final); e D4: 160 rãs $/ \mathrm{m}^{2}$ (inicial) e 75 rãs $/ \mathrm{m}^{2}$ (final). No ensaio de desempenho, foram utilizadas 558 rãs com peso médio de $26,80 \mathrm{~g}$ durante 56 dias, distribuídas em um delineamento em blocos casualizados, com seis repetições. Houve efeito das densidades sobre o ganho de peso, que foram inversamente proporcionais. Menores densidades resultaram em maior consumo de alimento, de modo que, com o uso das densidades iniciais de 75 e 105 rãs $/ \mathrm{m}^{2}$, obteve-se maior consumo de alimento que o uso de 125 e 160 rãs $/ \mathrm{m}^{2}$. A conversão alimentar aparente também variou; a densidade inicial de 160 rãs $/ \mathrm{m}^{2}$ proporcionou a pior média desse parâmetro. Os tratamentos foram avaliados economicamente, segundo os custos com alimentação, a receita bruta e a receita líquida parcial proporcionados. Concluiu-se que a densidade final de 50 rãs $/ \mathrm{m}^{2}$ aumentou a receita líquida parcial, proporcionando resultados mais adequados para produção de rãs.
\end{abstract}

Palavras-chave: avaliação econômica, pós-metamorfose, ranicultura

\section{Effects of Stocking Density on Performance of Bullfrog (Rana catesbeiana) in the Post-metamorphic Phase}

RESUMO - This work was carried out with the objective of evaluate four stocking densities of bullfrog (post-metamorphic) in mini-stalls. The densities studied were D1: 75 bullfrogs $/ \mathrm{m}^{2}$ (initial) and 35 bullfrogs $/ \mathrm{m}^{2}$ (finishing); D2: 105 bullfrogs $/ \mathrm{m}^{2}$ (initial) and 50 bullfrogs $/ \mathrm{m}^{2}$ (finishing); D3: 125 bullfrogs $/ \mathrm{m}^{2}$ (initial) and 60 bullfrogs $/ \mathrm{m}^{2}$ (finishing); and D4: 160 bullfrogs $/ \mathrm{m}^{2}$ (initial) and 75 bullfrogs $/ \mathrm{m}^{2}$ (finishing). The performance trial was carried out with 558 bullfrogs averaging initial weight of $26.80 \mathrm{~g}$, during 56 days, according to a completely randomized blocks design with six replicates. Inversely proportional effects of densities on weight gain were observed. Smaller densities resulted in greater values of feed intake, and the initial densities of 75 and 105 bullfrogs $/ \mathrm{m}^{2}$ resulted in higher values of feed intake than 125 and 160 bullfrogs $/ \mathrm{m}^{2}$. The apparent feed conversion also varied, showing the worst average on initial density of 160 bullfrogs $/ \mathrm{m}^{2}$. Economic analysis was performed with the costs with feeding, gross income and partial net income. It was concluded that the final density of 50 bullfrogs $/ \mathrm{m}^{2}$ increased the partial net income and was indicated for production of bullfrogs.

Key Words: bullfrog culture, economic evaluate

\section{Introdução}

A ranicultura vem despertando grande interesse de produtores e pesquisadores por ser uma atividade ainda pouco explorada, mas de grande potencial econômico. Atualmente, o Brasil é um dos maiores produtores do mundo de rãs criadas em cativeiro (Lima et al., 1999), mas demonstra estar ainda em fase de consolidação tecnológica (Lima et al., 2003), tornando fundamental a participação dos pesquisadores em trabalhos que envolvam manejo de criação.

Algumas vantagens podem ser obtidas com o uso de diferentes densidades de estocagem: densidades menores aumentam o tamanho e peso individual dos animais (Piedras \& Pouey, 2000; Hayashi et al., 2004;
Barcellos et al., 2004; Savolainen et al., 2004), enquanto densidades maiores elevam a produtividade por área (Stringhini et al., 1998; Oliveira et al., 2000; Brandão et al., 2004). Outros autores mostraram que o uso de densidades inadequadas pode resultar em efeitos negativos, prejudicando a produção e a qualidade do produto final (Lefrançois et al., 2001; Oliveira \& Carvalho, 2002; Yousif, 2002; Garcia et al., 2002).

Segundo Luz \& Zaniboni Filho (2002), a densidade de estocagem a ser utilizada depende da espécie, das condições de cultivo, do tipo de alimentação, do manejo adotado e do tamanho dos animais, entre outros fatores.

Lana et al. (2001) alertaram que a crescente pressão do mercado pode levar os criadores a elevarem

\footnotetext{
${ }^{1}$ Parte do trabalho do primeiro autor, como bolsista DCR, junto à UFPB - Bolsa CNPq.

2 Mestre em Zootecnia - UFPB (apcparaiba@yahoo.com.br).

3 Professor do Departamento de Tecnologia Rural/CFT/UFPB (moura321@zaz.com.br).

${ }^{4}$ Bolsista de Iniciação Científica - CNPq/UFPB (richelita.mendes@gmail.com).

5 Técnico em Agroindústria - CFT/UFPB (valnir.mc@bol.com.br).
} 
a taxa de lotação, como forma de reduzir os custos, mas, na maioria das vezes, isso tem sido feito sem a necessária readequação de equipamentos, nutrição e manejo ambiental à nova situação.

Dessa forma, esse tema foi alvo de várias pesquisas visando avaliar as melhores densidades de estocagem para diferentes espécies animais, em seu ambiente de criação, como aves em galpões (Mortari et al., 2002), suínos em baias (Banhazi et al, 2001), coelhos em gaiolas (Oliveira \& Almeida, 2002), camarões em viveiros (Zimmerman \& Raupp, 1992) e peixes em tanques e viveiros (Carneiro et al., 1999; Graeff \& Pruner, 1999 e 2000; Vieira et al., 2000; Gomes \& Schlindwein, 2000; Souza-Filho \& Cerqueira, 2003; Cavero et al., 2003; Salaro et al., 2003). Embora esses estudos tenham sido realizados com espécies diferentes, várias conclusões devem ser verificadas e avaliadas na recria de rãs, observando-se as devidas particularidades.

O estudo do efeito da densidade de estocagem em ranicultura se concentrou em pesquisas com animais na fase de girino (Soares et al., 1983; Justo et al., 1985; Flores-Nava \& Vera-Muñoz, 1999; Castro \& Pinto, 2000; Hayashi et al., 2004), que são criados em ambientes totalmente diferentes aos de recria.

Existem poucos trabalhos acerca do efeito da densidade no desempenho de rãs em recria (pósmetamorfose), e esses estudos trouxeram resultados quanto às taxas de mortalidade. Fontanello et al. (1987) avaliaram a influência da densidade de 5, 25, 45 e 65 rãs $/ \mathrm{m}^{2}$ na sobrevivência de rã-touro em recria e concluíram que, à medida que se aumentava a densidade, elevava-se a mortalidade. Rodriguez-Serna et al. (1996) estudaram os efeitos da densidade, em um sistema vertical de recria, e não encontraram diferenças entre os tratamentos com 50 e 100 rãs $/ \mathrm{m}^{2}$, enquanto aquele com 200 rãs $/ \mathrm{m}^{2}$ foi interrompido pela alta taxa de mortalidade.

O estudo dos custos de produção ou da viabilidade econômica, de propostas para aumento do desempenho animal é de fundamental importância para a recomendação do melhor tratamento, pois nem sempre o melhor resultado experimental se concretiza em melhor custo-benefício. Essa preocupação orientou pesquisadores na aréa, como Graeff et al. (2001) e Souza et al. (2003), em trabalhos realizados com peixes, e Fontanello et al. (1993), com rãs.

Objetivou-se com este trabalho comparar o desempenho de rãs em recria sob quatro densidades de estocagem e avaliá-las economicamente.

\section{Material e Métodos}

O ensaio foi conduzido no Setor de Ranicultura do CFT - Campus de Bananeiras da UFPB.

Foram avaliadas quatro densidades de estocagem (D1, D2, D3 e D4): D1: 75 rãs $/ \mathrm{m}^{2}$ (inicial) e 35 rãs $/ \mathrm{m}^{2}$ (final); D2: 105 rãs $/ \mathrm{m}^{2}$ (inicial) e 50 rãs $/ \mathrm{m}^{2}$ (final); D3: 125 rãs $/ \mathrm{m}^{2}$ (inicial) e 60 rãs $/ \mathrm{m}^{2}$ (final); e D4: 160 rãs $/ \mathrm{m}^{2}$ (inicial) e 75 rãs $/ \mathrm{m}^{2}$ (final). As densidades variaram durante o crescimento das rãs, em função das diferentes necessidades que esses animais apresentam por espaço e para manutenção das condições higiênicas da baia (Lima \& Agostinho, 1992), mantendo-se constante a relação animal por área de cocho entre os tratamentos.

Foram utilizadas 558 rãs-touro com peso médio

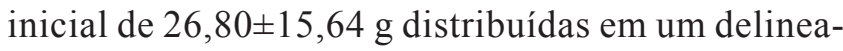
mento em blocos casualizados com seis repetições. Os blocos foram constituídos segundo a idade/peso dos animais, a fim de que o efeito desse parâmetro fosse isolado na análise de variância, de modo que o bloco de menor peso inicial apresentava tinha 7,2 $\pm 0,27 \mathrm{~g}$ e o de maior peso inicial, $45,5 \pm 1,48 \mathrm{~g}$.

As rãs foram alojadas em 24 mini-baias $\left(0,055 \mathrm{~m}^{3}\right.$ cada), formadas por caixas plásticas de tamanho fixo e piso de fibra de vidro, instaladas em uma sala de experimentação (Casali, 2003). As mini-baias possuíam abrigo, cocho e piscina e forneciam $0,2 \mathrm{~m}^{2}$ de área interna e $12 \mathrm{~L}$ de água (da piscina) por unidade experimental. O cocho, elaborado a partir de cano de PVC, possuía largura fixa e diferentes medidas de comprimento e ocupava locais estratégicos do ambiente, para manter a linearidade entre os elementos da baia (cocho, abrigo e piscina), conforme o modelo, em escala reduzida, do Sistema Anfigranja, proposto por Lima \& Agostinho (1992). Assim, o tamanho do cocho, em cada tratamento, foi: D1:25 cm; D2: $35 \mathrm{~cm}$; D3: $42 \mathrm{~cm}$ e D4: $54 \mathrm{~cm}$. Como foi necessário manter a mesma relação de área de cocho por animal e a linearidade dos elementos da baia, não foi possível manter eqüidistantes as densidades estudadas.

As temperaturas de mínima e máxima, na sala experimental, foram, respectivamente, de 21,0 e $31,0^{\circ} \mathrm{C}$. A média das temperaturas mínimas foi de $22,9^{\circ} \mathrm{C}$ e das máximas, de $27,5^{\circ} \mathrm{C}$, enquanto a média geral foi de $25,2^{\circ} \mathrm{C}$. As temperaturas coletadas na água da piscina apresentaram o menor valor observado de $21,5^{\circ} \mathrm{C}$ e o maior de $30,5^{\circ} \mathrm{C}$; a média das manhãs foi de $23,0^{\circ} \mathrm{C}$ e a das tardes, de $25,6^{\circ} \mathrm{C}$, ao passo que a média geral foi de $24,3^{\circ} \mathrm{C}$. 
Como atrativo alimentar, foram utilizadas larvasde-mosca (Musca domestica), que movimentavam as rações em proporção fixa de $20 \%$ (em peso) do alimento fornecido nos primeiros 28 dias de experimento, quando foi adotada a densidade inicial, e 10\%, nos 28 dias restantes, quando utilizada a densidade final. Portanto, o período experimental teve duração de 56 dias.

As rãs foram alimentadas ad libitum, apenas uma vez ao dia, com ração comercial para peixes carnívoros, contendo os seguintes níveis de garantia: 45\% (mín.) proteína bruta $(\mathrm{PB}), 14 \%$ (mín.) extrato etéreo (EE), 5\% (mín.) fibra bruta (FB), 14\% (máx.) matéria mineral (MM); e 87\% (mín.) matéria seca (MS). Durante o manejo, foram realizadas a alimentação, a limpeza das baias e a troca total da água das piscinas.

Os índices zootécnicos estudados foram o ganho de peso diário (GP), o consumo de alimento diário (ração e larvas) (CRL) e a conversão alimentar aparente (CAA). Para o cálculo do CRL e da CAA, foram descontadas as sobras de alimento no cocho (diariamente), mas não foi possível quantificar o desperdício de alimento espalhado na baia pelos animais. Ao final do período experimental, os animais foram pesados, utilizando-se balança eletrônica digital, com precisão de $0,01 \mathrm{~g}$. Os dados foram submetidos à análise de variância e as médias, comparadas pelo teste Student Newman-Keuls (SNK), a 5\% de probabilidade, pelo programa SAEG - Sistemas de Análises Estatísticas e Genéticas (UFV, 1997).

Avaliou-se economicamente o experimento segundo metodologia descrita por Souza et al. (2003), considerando-se apenas o custo da alimentação, que foi o fator variável, uma vez que os outros fatores geradores de custo, inclusive mão-de-obra, foram entendidos como constantes e iguais para os tratamentos analisados. O custo da alimentação foi calculado com base apenas no preço e no consumo da ração comercial: custo de alimentação $[\mathrm{C}=\mathrm{Qr} \times \operatorname{Pr}$, em que: Qré a quantidade de ração gasta e Pr, o preço do quilo da ração, cotado a $\mathrm{R} \$ 1,65]$. Calculou-se a receita bruta $[\mathrm{RB}=\mathrm{B} \times \mathrm{Pp}$, em que: B é a biomassa $(\mathrm{kg})$ e Pp, o preço $(\mathrm{R} \$)$ do quilograma de rã viva, cotado a $\mathrm{R} \$ 6,00]$ e a receita líquida parcial [ $\mathrm{RLP}=\mathrm{RB}-\mathrm{C}$ ], para as condições deste experimento, em um ciclo de produção, considerando a densidade final. Foi feita a projeção dos valores para produção em $1.000 \mathrm{~m}^{2} / \mathrm{ano}$, considerando toda área como baias de recria. A produção em um ano foi estimada a partir do número de safras por ano obtido em cada tratamento, tendo-se como peso referência para o abate $180 \mathrm{~g}$.

\section{Resultados e Discussão}

Na Tabela 1, estão apresentados os resultados de ganho de peso diário, consumo de alimento e conversão alimentar aparente, em função das diferentes densidades de estocagem.

Houve diferença estatística entre todas as densidades de estocagem estudadas, que foram inversamente proporcionais ao ganho de peso (Tabela 1). A densidade inicial de 75 rãs $/ \mathrm{m}^{2}$ proporcionou maior valor $(\mathrm{P}<0,05)$ de ganho de peso diário $(2,18 \mathrm{~g})$ que a $105 \mathrm{rãs} / \mathrm{m}^{2}$, que, por sua vez, foi melhor que a de 125 rãs $/ \mathrm{m}^{2}$. A densidade de 160 rãs $/ \mathrm{m}^{2}$ resultou em menor ganho de peso diário. Apenas os animais da densidade inicial de 75 rãs $/ \mathrm{m}^{2}$ e pertencentes aos blocos de maior peso inicial atingiram o ponto de abate, com peso médio final superior a $180 \mathrm{~g}$.

O ganho de peso por dia, em todos os tratamentos, foi maior que os citados por Lima \& Agostinho (1988), que constataram $0,88 \mathrm{~g}$ de ganho de peso, em animais com peso inicial de $36,6 \mathrm{~g}$. Resultado semelhante foi descrito por Lima et al. (1988), que observaram 0,84 $\mathrm{g}$ de ganho de peso, em animais com peso próximo ao do trabalho anterior. Lima et al. (2003), coletando dados de ranários onde se adotavam densidades de 50 rãs $/ \mathrm{m}^{2}$, descreveram ganhos de peso de 1,2 g para o período de crescimento ( 40 a $100 \mathrm{~g}$ ) e de $1,7 \mathrm{~g}$ para o período de terminação (100 a 200 g), ao passo que Braga \& Lima (2001), utilizando densidade aproximada de 20 rãs $/ \mathrm{m}^{2}$, encontraram ganho de peso médio de $1,50 \mathrm{~g}$, em animais com peso inicial de 37,1 g. Rodriguez-Serna et al. (1996) obtiveram resultados de ganho de peso por dia, nas densidades de $50 \mathrm{rãs} / \mathrm{m}^{2} \mathrm{e}$ 100 rãs $/ \mathrm{m}^{2}$, de 0,67 e $0,60 \mathrm{~g}$, respectivamente, demonstrando valores menores que os deste experimento.

O mesmo comportamento de queda de desempenho dos animais em função do aumento da densidade foi observado em peixes por Piedras \& Pouey (2000), Vieira et al. (2000), Graeff \& Pruner (2000), Graeff et al. (2001), Yousif (2002), Luz \& Zaniboni Filho (2002), Barcellos et al. (2004) e Savolainen et al. (2004). Do mesmo modo, para girinos de rã-touro, Hayashi et al. (2004) confirmaram os resultados encontrados por esses autores. Em contraposição, Rodriguez-Serna et al. (1996) não verificaram diferença, ao utilizarem 50 e 100 rãs $/ \mathrm{m}^{2}$ na recria.

Menores valores de densidades resultaram em maiores consumos de alimento diário (Tabela 1). Com o uso das densidades iniciais de estocagem de 75 e 105 rãs $/ \mathrm{m}^{2}$, iguais estatisticamente, obteve-se maior 
Tabela 1 - Valores médios de ganho de peso diário (GPD), consumo de alimento diário (CRL) e conversão alimentar aparente (CAA) de rã-touro aos 56 dias, em função de diferentes densidades de estocagem

Table 1 - Mean values of daily weight gain (DWG), daily feed intake (DFI) and apparent feed conversion (AFC) of bullfrogs at 56 days on different stocking densities

\begin{tabular}{|c|c|c|c|}
\hline $\begin{array}{l}\text { Densidade } \\
\text { Density }\end{array}$ & $\begin{array}{l}\mathrm{GPD}(\mathrm{g} / \mathrm{d}) \\
D W G\end{array}$ & $\begin{array}{l}\mathrm{CRL}(\mathrm{g}) \\
\text { DFI }\end{array}$ & $\begin{array}{l}\text { CAA } \\
A F C\end{array}$ \\
\hline $\begin{array}{l}75 \text { rãs } / \mathrm{m}^{2} \text { (inicial) e } 35 \text { rãs } / \mathrm{m}^{2} \text { (final) } \\
75 \text { bullfrogs } / \mathrm{m}^{2} \text { (initial) and } 35 \text { bullfrogs } / \mathrm{m}^{2} \text { (final) }\end{array}$ & $2,18 \mathrm{a}$ & $3,44 a$ & $1,62 \mathrm{a}$ \\
\hline $\begin{array}{l}105 \mathrm{rãs} / \mathrm{m}^{2} \text { (inicial) e } 50 \text { rãs } / \mathrm{m}^{2} \text { (final) } \\
105 \text { bullfrogs } / \mathrm{m}^{2} \text { (initial) and } 50 \text { bullfrogs } / \mathrm{m}^{2} \text { (final) }\end{array}$ & $1,99 b$ & $3,63 a$ & $1,82 \mathrm{a}$ \\
\hline $\begin{array}{l}125 \text { rãs } / \mathrm{m}^{2} \text { (inicial) e } 60 \text { rãs } / \mathrm{m}^{2} \text { (final) } \\
125 \text { bullfrogs } / \mathrm{m}^{2} \text { (initial) and } 60 \text { bullfrogs } / \mathrm{m}^{2} \text { (final) }\end{array}$ & $1,54 \mathrm{c}$ & $2,61 b$ & $1,83 \mathrm{a}$ \\
\hline $\begin{array}{l}160 \text { rãs } / \mathrm{m}^{2} \text { (inicial) e } 75 \text { rãs } / \mathrm{m}^{2} \text { (final) } \\
160 \text { bullfrogs } / \mathrm{m}^{2} \text { (initial) and } 75 \text { bullfrogs } / \mathrm{m}^{2} \text { (final) }\end{array}$ & $1,38 \mathrm{~d}$ & $2,91 b$ & $2,20 \mathrm{~b}$ \\
\hline CV $(\%)$ & 5,62 & 8,33 & 10,05 \\
\hline
\end{tabular}

$a, b, c$ Médias seguidas das mesmas letras, na mesma coluna, não diferem $(P>0,05)$ pelo teste SNK.

a, $b, c$ Means with same letters, in the column, do not differ $(P>0.05)$ by SNK test.

$(\mathrm{P}<0,05)$ consumo de alimento em relação a 125 e 160 rãs $/ \mathrm{m}^{2}$, também iguais entre si. O consumo de alimento, em todos os tratamentos, foi menor que o encontrado por Lima et al. (1988) de 5,18 g, para animais com peso inicial de 37,44 g até 90 dias de criação. Entretanto, o maior consumo observado por esses autores não resultou em ganho de peso maior, e sim em pior conversão alimentar. Menores valores de consumo de alimento foram observados em ranários comerciais que utilizaram a densidade de $50 \mathrm{rãs} / \mathrm{m}^{2}$, por Lima et al. (2003), de 1,56 g, para o período de crescimento (40 a 100 g), e de 2,38 g, para o período de terminação (100 a 200 g). Neste caso, os menores consumos de alimento observados pelos autores supracitados, corresponderam a melhores resultados de conversão alimentar. Braga \& Lima (2001), utilizando a densidade de 20 rãs $/ \mathrm{m}^{2}$, também encontraram menor consumo de alimento $(1,82 \mathrm{~g})$ em rãs com peso inicial de $37,1 \mathrm{~g}$, em 35 dias de experimento.

Comportamento inversamente proporcional entre densidade de estocagem e consumo de alimento também foi verificado em peixes por Padua et al. (1998) e Cavero et al. (2003).

A conversão alimentar aparente foi influenciada $(\mathrm{P}<0,05)$ com o uso das diferentes densidades de estocagem (Tabela 1): a densidade inicial de 160 rãs $/ \mathrm{m}^{2}$ proporcionou a pior média desse parâmetro $(2,20: 1)$, em relação às demais, estatisticamente iguais entre si.

Os resultados de conversão alimentar aparente média deste trabalho são melhores que os citados por Lima et al. (1988), que encontraram 6,17:1, possivelmente em razão das diferenças de instalações, rações e manejo alimentar. Observa-se semelhança de resul- tados com o uso de densidades de 50 rãs $/ \mathrm{m}^{2}$ em ranários comerciais: Lima et al. (1997) encontraram valores de conversão alimentar de 1,46:1 e Lima et al. (2003) reportaram relações de 1,3:1, para a fase de crescimento (40 a $100 \mathrm{~g}$ ), e de 1,4:1, para a fase de terminação (100 a 200 g). Provavelmente, além das diferenças entre as rações fornecidas, a necessidade de controle de desperdícios em ranários comerciais e de alimentação ad libitum em experimentos tenha influenciado os diferentes resultados. Braga \& Lima (2001), utilizando baixa densidade de $20 \mathrm{rãs} / \mathrm{m}^{2}$, em laboratório com temperatura controlada, também encontraram melhor conversão alimentar média de 1,22:1.

Aumento das médias de conversão alimentar, em função do aumento da densidade, foi verificado em carpa comum por Graeff et al. (2001), mas não foi encontrado por outros autores em estudos com peixes (Padua et al., 1998; Gomes \& Schlindwein, 2000; Cavero et al., 2003; Brandão et al., 2004; Barcellos et al., 2004). Hayashi et al. (2004) não constataram diferença para conversão alimentar, ao estudarem densidade de girinos. Peixes e girinos são criados em ambiente totalmente aquático, o que difere da recria de rãs. Provavelmente, o aumento da conversão alimentar aparente foi causado pelo maior trânsito de animais, causado pelo estresse da alta densidade, e conseqüente desperdício de ração na água. O acúmulo de ração na água e a maior quantidade de fezes, observados durante o experimento nos tratamentos de alta densidade podem ter sido uma das causas do baixo desempenho dos animais, piorando as condições higiênicas da baia, como relatado por Lima \& Agostinho (1992), que descreveram os prejuízos decorren- 
tes da alta densidade. Portanto, recomendam-se novas pesquisas com manejo de troca de água, para manutenção da qualidade do ambiente da piscina, em altas densidades, para verificar se as rãs respondem com melhor desempenho.

Em todo o período experimental (56 dias), morreram apenas três animais, correspondendo a $0,63 \%$ do lote de rãs utilizadas, apresentando comportamento aleatório. Dessa forma, o parâmetro mortalidade foi desconsiderado para comparações entre as densidades.

Constam na Tabela 2 os resultados da análise econômica do experimento, de acordo com as diferentes densidades avaliadas.

Para efeito de comparação, considerou-se o melhor valor obtido em cada variável estudada como equivalente a $100 \%$ em relação aos demais tratamentos, nas discussões seguintes.

Analisando apenas um ciclo de produção e a quantidade de animais utilizada neste experimento, verificouse que a menor densidade final $\left(35 \mathrm{rãs} / \mathrm{m}^{2}\right)$ proporcionou custos com alimentação menores 50,85; 30,07 e 81,27\% que as densidades de 50, 60 e 75 rãs $/ \mathrm{m}^{2}$, respectivamente.

Em compensação, verificou-se, ao analisar a receita bruta, que a maior densidade final ( $\left.75 \mathrm{rãs} / \mathrm{m}^{2}\right)$ resultou em maiores receitas brutas, que foram 26,28 ; 4,01 e 10,72\% superiores às observadas nas densidades de 35, 50 e 60 rãs $/ \mathrm{m}^{2}$ respectivamente. Esses resultados proporcionaram diferentes receitas líquidas, apesar de estarem próximas. A densidade de 50 rãs $/ \mathrm{m}^{2}$ ocasionou ganhos maiores $(12,58 ; 0,14 \mathrm{e}$ $11,99 \%$ ) que as densidades de 35,60 e 75 rãs $/ \mathrm{m}^{2}$, respectivamente. Portanto, a maior receita bruta resultante do aumento da densidade foi prejudicada pelas piores conversões alimentares obtidas com o uso de altas densidades, resultando em aumento dos custos com alimentação.

Analisando a previsão feita quando se aumenta a área de produção, ocorrendo diferentes números de safras, de acordo com o maior ou menor ganho de peso dos animais, para o período de um ano, verificaram-se modificações nos resultados. A densidade de 60 rãs $/ \mathrm{m}^{2}$ proporcionou custo de alimentação menores $(8,84 ; 49,65$ e 24,89\%) que as densidades de 35, 50 e 75 rãs $/ \mathrm{m}^{2}$, respectivamente.

Verificou-se que a densidade de 50 rãs $/ \mathrm{m}^{2}$ resultou em receita bruta 15,$74 ; 27,92$ e $27,65 \%$ maior que as densidades de 35,60 e 75 rãs $/ \mathrm{m}^{2}$, respectivamente, ou seja, o aumento de densidade elevou a receita bruta apenas para o tratamento de 50 rãs $/ \mathrm{m}^{2}$. Assim, a análise da receita líquida parcial apresentou resultado diferente quando comparado a apenas um ciclo de produção, como era esperado. Novamente, destacou-se densidade de 50 rãs $/ \mathrm{m}^{2}$, que proporcionou ganhos 4,09; 22,60 e 38,87\% maiores que as densidades de 35,60 e 75 rãs $/ \mathrm{m}^{2}$, respectivamente. Portanto, ao prever produção anual para $1.000 \mathrm{~m}^{2}$, menores densidades de estocagem aumentaram as receitas líquidas.

Provavelmente, ao se trabalhar com altas densidades, sejam necessários métodos para reduzir o desperdício de ração e manter a qualidade da água da piscina. Nesse caso, os resultados com densidades acima de 50 rãs $/ \mathrm{m}^{2}$ talvez sejam melhores.

Tabela 2 - Valores médios obtidos em reais $(R \$)^{1}$ para custo com alimentação $(C)$, receita bruta (RB) e receita líquida parcial (RLP) na recria de rã-touro, em cada ciclo de produção (1,2 $\mathrm{m}^{2}$ por tratamento) e valores projetados em $1.000 \mathrm{~m}^{2} /$ ano

Table 2 - Mean values of costs with feeding (FC), gross income (GI) and partial net income (PNI,) in the bullfrog post metamorphic stage, during each cycle of production $\left(1.2 \mathrm{~m}^{2}\right.$ by treatment) and the expected values in 1,000 $\mathrm{m}^{2} /$ year

\begin{tabular}{|c|c|c|c|c|c|c|}
\hline \multirow[t]{2}{*}{$\begin{array}{l}\text { Densidade } \\
\text { Density }\end{array}$} & \multicolumn{3}{|c|}{$\begin{array}{l}\text { Valores por ciclo de produção }\left(1,2 \mathrm{~m}^{2}\right) \\
\text { Values from each cycle of production }\end{array}$} & \multicolumn{3}{|c|}{$\begin{array}{l}\text { Valores projetados para } 1.000 \mathrm{~m}^{2} / \text { ano } \\
\text { Expected values for } 1,000 \mathrm{~m}^{2} \text { year }\end{array}$} \\
\hline & $\begin{array}{c}\mathrm{C} \\
F C\end{array}$ & $\begin{array}{l}\mathrm{RB} \\
G I\end{array}$ & $\begin{array}{l}\text { RLP } \\
P N I\end{array}$ & $\begin{array}{c}\mathrm{C} \\
F C\end{array}$ & $\begin{array}{l}\mathrm{RB} \\
G I\end{array}$ & $\begin{array}{l}\text { RLP } \\
P N I\end{array}$ \\
\hline $\begin{array}{l}35 \text { rãs } / \mathrm{m}^{2} \text { (final) } \\
35 \text { bullfrogs } / m^{2} \text { (final) }\end{array}$ & 66,75 & 153,82 & 87,07 & $245.892,52$ & $566.644,49$ & $320.751,97$ \\
\hline $\begin{array}{l}50 \text { rãs } / \mathrm{m}^{2} \text { (final) } \\
50 \text { bullfrogs } / \mathrm{m}^{2} \text { (final) }\end{array}$ & 100,69 & 200,29 & 99,60 & $338.103,76$ & $672.521,54$ & $334.417,78$ \\
\hline $\begin{array}{l}60 \text { rãs } / \mathrm{m}^{2} \text { (final) } \\
60 \text { bullfrogs } / \mathrm{m}^{2} \text { (final) }\end{array}$ & 86,82 & 186,28 & 99,46 & $225.930,47$ & $484.755,04$ & $258.824,57$ \\
\hline $\begin{array}{l}75 \text { rãs } / \mathrm{m}^{2} \text { (final) } \\
75 \text { bullfrogs } / m^{2} \text { (final) }\end{array}$ & 121,00 & 208,66 & 87,66 & $282.160,15$ & $486.574,20$ & $204.414,05$ \\
\hline
\end{tabular}




\section{Conclusões}

O aumento da densidade de estocagem diminuiu o ganho de peso diário e piorou a conversão alimentar aparente das rãs.

Com base na análise econômica, verificou-se que o uso de densidades finais acima de $50 \mathrm{rãs} / \mathrm{m}^{2}$ não aumentou a receita bruta suficientemente para compensar as perdas em ganho de peso.

A densidade de estocagem final de $50 \mathrm{rãs} / \mathrm{m}^{2}$ elevou a receita líquida parcial, apresentando resultados mais adequados para produção de rãs.

\section{Literatura Citada}

BANHAZI, T.; HARPER, C.C.Z.; GLATZ, P. The effect of stocking rate on pig production. In: AGRIBUILDING 2001 SYMPOSIUM, Campinas. Abstracts... Campinas: Embrapa Suínos e Aves, 2001. p.19-20.

BARCELLOS, L.J.G.; KREUTZ, L.C.; QUEVEDO, R.M. et al. Nursery rearing of jundiá, rhamdia quelen (quoy \& gaimard) in cages: cage type, stocking density and stress response to confinement. Aquaculture, v.232, n.1-4, p.383 - 394, 2004.

BRAGA, L.G.T.; LIMA, S.L. Influência da temperatura ambiente no desenvolvimento rã-touro, Rana catesbeiana (Shaw, 1802) na fase de recria. Revista Brasileira de Zootecnia, v.30, n.6, p.1659-1663, 2001.

BRANDÃO, F.R.; GOMES, L.C.; CHAGAS, E.C. et al. Densidade de estocagem de juvenis de tambaqui durante a recria em tanques-rede. Pesquisa Agropecuária Brasileira, v.39, n.4, p.357-362, 2004.

CARNEIRO, P.C.F.; CYRINO, J.E.P.; CASTAGNOLLI, N. Produção da tilápia vermelha da Flórida em tanques-rede. Scientia Agricola, v.56, n.3, p.673-679, 1999.

CASALI, A. P. Avaliação de rações comerciais na recria de rã-touro (Rana catesbeiana, Shaw 1802) em mini-baias. Areia: Universidade Federal de Paraíba, 2003. 87p. (Mestrado em Zootecnia) - Universidade Federal de Paraíba, 2003.

CASTRO, J.C.; PINTO, A.T. Qualidade de água em tanques de girinos de rã-touro, Rana catesbeiana, Shaw, 1802, cultivadas em diferentes densidades de estocagem. Revista Brasileira de Zootecnia, v.29, n.6, p.1903-1911, 2000

CAVERO, B.A.S.; PEREIRA-FILHO, M.; ROUBACH, R. et al. Efeito da densidade de estocagem na homogeneidade do crescimento de juvenis de pirarucu em ambiente confinado. Pesquisa Agropecuária Brasileira, v.38, n.1, p.103-107, 2003.

FLORES-NAVA, A.; VERA-MUÑOZ, P. Growth, metamorphosis and feeding behavior of Rana catesbeiana Shaw 1802 tadpoles at different rearing densities. Aquaculture Research, v.30, n.5, p.341-347, 1999.

FONTANELLO, D.; SOARES, H.A.; MANDELI JR., J. et al. Influência da densidade populacional na sobrevivência de rãtouro (Rana catesbeiana Shaw, 1802) em criação intensiva. Brazilian Journal of Veterinary Research and Animal Science, v.24, n.2, p.213-216, 1987.

FONTANELLO, D.; WIRZ, R.R.; SOARES, H.A. et al. Comparação de quatro sistemas de engorda de rãs-touro (Rana catesbeiana Shaw, 1802): tanque-ilha, confinamento, anfigranja e gaiolas. 1. Desenvolvimento ponderal; 2. Custo operacional. Boletim do Instituto de Pesca, v.20 (único), p.43-58, 1993.

GARCIA, R.G.; MENDES, A.A.; GARCIA, E.A. et al. Efeito da densidade de criação e do sexo sobre o empenamento, incidência de lesões na carcaça e qualidade da carne de peito de frangos de corte. Revista Brasileira de Ciência Avícola, v.4, n.1, p.27-35, 2002.

GRAEFF, A.; PRUNER, E.N. Efeito da densidade de estocagem na produtividade final de carpas, Cyprinus carpio Linnaeus, 1758 (var. Specularis) na fase de engorda. Período - inverno. Ciência e Agrotecnologia, v.23, n.4, p.958-967, 1999.

GRAEFF, A.; PRUNER, E.N. Efeito da densidade de povoamento na produtividade final em carpas (Cyprinus carpio var. Specularis) em fase de engorda, durante o verão. Revista Brasileira de Zootecnia, v.29, n.3, p.639-645, 2000.

GRAEFF, A.; KREUZ, C.L.; PRUNER, E.N. et al. Viabilidade econômica de estocagem de alevinos de carpa comum (Cyprinus Carpio var. Specularis) no inverno em alta densidade. Revista Brasileira de Zootecnia, v.30, n.4, p.1150-1158, 2001.

GOMES, S.Z.; SCHLINDWEIN, A.P. Efeito de períodos de cultivo e densidades de estocagem sobre o desempenho do catfish (Ictalurus punctatus) nas condições climáticas do litoral de Santa Catarina. Revista Brasileira de Zootecnia, v.29, n.5, p.1266-1272, 2000.

HAYASHI, C.; SOARES, C.M.; GALDIOLI, E.M. et al. Desenvolvimento de girinos de rã-touro (Rana catesbeiana, Shaw, 1802) cultivados em diferentes densidades de estocagem em tanques-rede. Revista Brasileira de Zootecnia, v.33, n.1, p.14-20, 2004.

JUSTO, C.L.; PENTEADO, L.A.; FONTANELLO, D. et al. Ganho de peso de girinos de Rana catesbeiana Shaw, 1802, em criação intensiva, sob diferentes densidades populacionais. Boletim do Instituo de Pesca, v.12, n.3, p.31-37, 1985.

LANA, G.R.Q.; SILVA JR., R.G.S.; VALERIO, S.R. et al. Efeito da densidade e de programas de alimentação sobre o desempenho de frangos de corte. Revista Brasileira de Zootecnia, v.30, n.4, p.1258-1265, 2001.

LEFRANÇOIS, C.; CLAIREAUXA, G.; MERCIERA, C. et al. Effect of density on the routine metabolic expenditure of farmed rainbow trout (Oncorhynchus mykiss). Aquaculture, v.195, p.269-277, 2001

LIMA, S. L.; AGOSTINHO, C.A. Índices de produtividade da rãtouro, Rana catesbeiana, na Anfigranja. In: ENCONTRO NACIONAL DE RANICULTURA - ENAR, 6., 1988, Rio de Janeiro. Coletânea... Rio de Janeiro: Associação dos Ranicultores do Estado do Rio de Janeiro - ARERJ, 1988. p.29-34.

LIMA, S.L.; AGOSTINHO, C.A.; PIRES, J.S.R. Ganho de peso e conversão alimentar da rã-touro, Rana catesbeiana, com ração (dados preliminares). In: ENCONTRO NACIONAL DE RANICULTURA - ENAR, 6., 1988, Rio de Janeiro. Coletânea... Rio de Janeiro: Associação dos Ranicultores do Estado do Rio de Janeiro - ARERJ, 1988. p.35-40.

LIMA, S.L.; AGOSTINHO, C.A. A tecnologia de criação de rãs. Viçosa, MG: Universidade Federal de Viçosa, 1992. 168p.

LIMA, S.L.; LANZA, A.L.L.; CASALI, A.P. et al. Sistema Anfigranja I: Índices de produtividade com rã touro, Rana catesbeiana SHAW, 1802. In: INTERNATIONAL MEETING ON FROG RESEARCH AND TECHLOLOGY, 2.: ENCONTRO NACIONAL DE RANICULTURA, 9., 1997, Santos. Anais... Santos: ABETRA, 1997. p.198.

LIMA, S.L.; CRUZ, T.A.; MOURA, O.M. Ranicultura: análise da cadeia produtiva. Viçosa, MG: Folha de Viçosa, 1999. 172p.

R. Bras. Zootec., v.34, n.6, p.1828-1834, 2005 
LIMA, S.L.; CASALI, A.P.; AGOSTINHO, C.A. Desempenho zootécnico e percentual de consumo de alimento de rã-touro (Rana catesbeiana) na fase de recria do sistema anfigranja. Revista Brasileira de Zootecnia, v.32, n.3, p.505-511, 2003.

LUZ, R.K.; ZANIBONI FILHO, E. Larvicultura do mandiamarelo Pimelodus maculatus Lacépède, 1803 (siluriformes: pimelodidae) em diferentes densidades de estocagem nos primeiros dias de vida. Revista Brasileira de Zootecnia, v.31, n.2, p.560-565, 2002.

MORTARI, A.C.; ROSA, A.P.; ZANELLA, I. et al. Desempenho de frangos de corte criados em diferentes densidades populacionais, no inverno, no sul do Brasil. Ciência Rural, v.32, n.3, p.493-497, 2002.

OLIVEIRA, M.C.; ALMEIDA, C.V. Desempenho de coelhos em crescimento criados em diferentes densidades populacionais. Arquivo Brasileiro de Medicina Veterinária e Zootecnia, v.54, n.5, p.530-533, 2002.

OLIVEIRA, J.E.; SAKOMURA, N.K.; FIGUEIREDO, A.N. et al. Efeito do isolamento térmico de telhado sobre o desempenho de frangos de corte alojados em diferentes densidades. Revista Brasileira de Zootecnia, v.29, n.5, p.14271434, 2000 .

OLIVEIRA, M.C.; CARVALHO, I.D. Rendimento e lesões em carcaça de frangos de corte criados em diferentes camas e densidades populacionais. Ciência Agrotécnica, v.26, n.5, p.1076-1081, 2002.

PIEDRAS, R.S.; POUEY, F.O.L.J. Efeito da densidade na sobrevivência e crescimento de larvas de peixe-rei de água doce (Odontesthes bonariensis). In: REUNIÃO ANUAL DA SOCIEDADE BRASILEIRA DE ZOOTECNIA, 37., 2000, Viçosa-MG. Anais... Viçosa, MG: SBZ, 2000. 1 CD-ROM.

PADUA, D.M.C.; SILVA, P.C.; PADUA, J.T. et al. Efeito da densidade de lotação e renovação da água no desenvolvimento do pacu, Piaractus mesopotamicu. Anais das Escolas de Agronomia e Veterinária. Goiânia: CEGRAF/UFG, v.28, n.1, p.43-53, 1998.

RODRÍGUEZ-SERNA, M.;FLORES-NAVA,A.;OLVERANOVOA, M.A. et al. Growth and production of bullfrog Rana catesbeiana Shaw, 1802, at three stocking densities in a vertical intensive culture system. Aquaculture Engineering, v.15, n.4, p.233-242, 1996.

UNIVERSIDADE FEDERAL DE VIÇOSA - UFV. SAEG Sistemas de análises estatísticas ( $\mathrm{v}$ 8.0) Viçosa, MG.

SALARO, A.L.; LUZ, R.K.; NOGUEIRA, G.C.C.B. et al. Densidades de estocagem na produção de alevinos de trairão (Hoplias cf. lacerdae). Revista Brasileira de Zootecnia, v.32, n.5, p.1033-1036, 2003.

SAVOLAINEN, R.; RUOHONEN, K.; RAILO, E. Injuries of stage 2 juvenile signal crayfish Pasifastacus leniusculus Dana. Aquaculture, v.231, n.1-4, p.237-248, 2004.
STRINGHINI, J.H.; ARIKI, J.; CAFÉ, M.B. et al. Níveis de lisina para frangos de corte criados em duas densidades populacionais. In: REUNIÃO ANUAL DA SOCIEDADE BRASILEIRA DE ZOOTECNIA, 35., 1998, Botucatu. Anais... Botucatu: Sociedade Brasileira de Zootecnia, 1998. p. 216.

SOARES, H.A.; FONTANELLO, D.; REIS, J.M. et al. Efeito da densidade de população no ganho de peso de girinos da rã touro (Rana catesbeiana Shaw, 1802). Boletim do Instituto de Pesca, v.10, n.único, p.47-51, 1983.

SOUZA, V.L.; URBINATI, E.C.; MARTINS, M.I.E.G. et al. Avaliação do crescimento e do custo da alimentação do pacu (Piaractus mesopotamicus Holmberg, 1887) submetido a ciclos alternados de restrição alimentar e realimentação. Revista Brasileira de Zootecnia, v.32, n.1, p.19-28, 2003.

SOUZA-FILHO, J.J.; CERQUEIRA, V.R. Influência da densidade de estocagem no cultivo de juvenis de robalo-flecha mantidos em laboratório. Pesquisa Agropecuária Brasileira, v.38, n.11, p.1317-1322, 2003.

UNIVERSIDADE FEDERAL DE VIÇOSA - UFV. SAEG Sistemas de análises estatísticas. Versão 7.1. Viçosa, MG: 1997. 150p (manual do usuário).

VIEIRA, J.S.; LOGATO, P.V.R.L.; FREITAS, R.T.F. et al. Efeito da densidade de estocagem no desempenho de tilápias do nilo (Oreochromis niloticus) em tanques-rede. In: REUNIÃO ANUAL DA SOCIEDADE BRASILEIRA DE ZOOTECNIA, 37., 2000, Viçosa, MG. Anais... Viçosa, MG: Sociedade Brasileira de Zootecnia, 2000. 1 CD-ROM.

YOUSIF, O.M. Emir. The effects of stocking density, water exchange rate, feeding frequency and grading on size hierarchy development in juvenile Nile tilapia, Oreochromis niloticus L. Emirates Journal of Agricultural Sciences, v.14, p.45-53, 2002.

ZIMMERMANN, S.; RAUPP, A.B. Cultivo do camarão de água doce, (Macrobrachium rosenbergii), em viveiros no sul do Brasil, sob diferentes densidades e taxas de estocagem. Pesquisa Agropecuária Brasileira, v.27, n.1, p.87-89, 1992. 